D. M. Ouwens $\cdot$ C. Boer $\cdot$ M. Fodor $\cdot$ P. de Galan •

\author{
R. J. Heine · J. A. Maassen - M. Diamant
}

\title{
Cardiac dysfunction induced by high-fat diet is associated with altered myocardial insulin signalling in rats
}

Received: 27 September 2004 / Accepted: 3 February 2005 / Published online: 30 April 2005

(C) Springer-Verlag 2005

\begin{abstract}
Aims/hypothesis: Diabetic cardiomyopathy (DCM) is common in type 2 diabetes. In DCM, insulin resistance may alter cardiac substrate supply and utilisation leading to changes in myocardial metabolism and cardiac function. In rats, exposure to excessive alimentary fat, inducing a type 2 diabetic phenotype, may result in myocardial insulin resistance and cardiac functional changes resembling DCM. Materials and methods: Rats received high-fat (HFD) or low-fat (LFD) diets for 7 weeks. Prior to killing, insulin or saline was injected i.p. Contractile function and insulin signalling were assessed in papillary muscles and ventricular lysates, respectively. Results: Fasting and post-load blood glucose levels were increased in HFD- vs LFD-rats (all $p<0.02$ ). Mean heart weight, but not body weight, was increased in HFD-rats $(p<0.01)$. HFD-hearts showed structural changes and triglyceride accumulation. HFD-muscles
\end{abstract}

D. M. Ouwens $(\bowtie) \cdot$ P. de Galan · J. A. Maassen

Department of Molecular Cell Biology,

Leiden University Medical Centre,

Wassenaarseweg 72 ,

2333 AL Leiden, The Netherlands

e-mail: d.m.ouwens@lumc.nl

Tel.: +31-71-5276296

Fax: +31-71-5276437

C. Boer

Laboratory for Physiology,

VU University Medical Centre,

Amsterdam, The Netherlands

M. Fodor

Department of Paediatrics,

VU University Medical Centre,

Amsterdam, The Netherlands

R. J. Heine · J. A. Maassen · M. Diamant

Department of Endocrinology/Diabetes Centre,

VU University Medical Centre,

Amsterdam, The Netherlands

M. Fodor

Department of Anatomy and Embryology,

Leiden University Medical Centre,

Leiden, The Netherlands developed higher baseline and maximum forces, but showed impaired recovery from higher workloads. Insulin-associated modulation of $\mathrm{Ca}^{2+}$-induced force augmentation was abolished in HFD-muscles. HFD reduced insulin-stimulated IRS1-associated phosphatidylinositol 3'-kinase activity and phosphorylation of protein kinase B, glycogen synthase kinase- $3 \beta$, endothelial nitric oxide synthase, and forkhead transcription factors by $40-60 \%$ (all $p<0.05$ ). Insulin-mediated phosphorylation of phospholamban, a critical regulator of myocardial contractility, was decreased in HFD-hearts $(p<0.05)$. Conclusions/interpretation: HFD induced a hypertrophy-like cardiac phenotype, characterised by a higher basal contractile force, an impaired recovery from increased workloads and decreased insulin-mediated protection against $\mathrm{Ca}^{2+}$ overload. Cardiac dysfunction was associated with myocardial insulin resistance and phospholamban hypophosphorylation. Our data suggest that myocardial insulin resistance, resulting from exposure to excessive alimentary fat, may contribute to the pathogenesis of diabetes-related heart disease.

Keywords Diabetes mellitus - Diabetic cardiomyopathy · Diet $\cdot$ Insulin $\cdot$ Phospholamban

Abbreviations DCM: diabetic cardiomyopathy - eNOS: endothelial nitric oxide synthase $\cdot \mathrm{F}_{\mathrm{dev}}$ : developed force . $\mathrm{F}_{\text {max }}$ : maximal force $\cdot \mathrm{F}_{\text {rec }}$ : recovered force $\cdot \mathrm{FOXO} / \mathrm{FKHR}$ : forkhead transcription factor-GSK3: glycogen synthase kinase 3 - HFD: high-fat diet - LFD: low-fat diet - mTOR: mammalian target of rapamycin - PI3K:

phosphatidylinositol 3'-kinase $\cdot \mathrm{PKB} / \mathrm{Akt}$ : protein kinase B - PLB: phospholamban - RyR: ryanodine receptor . SERCA2a: sarcoplasmic reticulum $\mathrm{Ca}^{2+}$ ATPase

\section{Introduction}

Type 2 diabetes is associated with a high incidence of coronary artery disease and congestive heart failure $[1,2]$. Even in asymptomatic patients with uncomplicated type 2 diabetes, left ventricular hypertrophy and dysfunction are 
found $[3,4]$. In the absence of coronary artery disease and hypertension, the myocardial abnormalities are ascribed to diabetic cardiomyopathy (DCM) [5]. Changes in myocardial energy metabolism, owing to altered cardiomyocyte substrate supply and utilisation, may underlie the development of DCM [6]. A key mechanism contributing to myocardial dysmetabolic changes may be impaired cardiac insulin signalling [6].

In insulin-resistant and type 2 diabetic subjects, high fluxes of NEFA and triglycerides exist because of unsuppressed lipolysis in adipose tissue and hepatic overproduction of triglyceride-rich particles. This excessive lipid exposure, in the presence of impaired glucose utilisation, results in accumulation of triglycerides in non-adipose tissues, including the myocardium. Inappropriate triglyceride deposition enlarges the intracellular pool of fatty acyl-CoA, thereby providing substrate for non-oxidative metabolic pathways leading to oxidative stress, cellular dysfunction and apoptosis [7]. In animal models of lipotoxicity, myocardial dysfunction was found, which was reversed by antisteatotic treatment [8]. In vitro studies in cardiomyocytes from Zucker fatty rats suggest that impaired cardiac insulin signalling may underlie the observed abnormalities [9]. These findings are mainly derived from studies in genetic models of insulin resistance and type 2 diabetes, and therefore, it is unclear whether similar mechanisms operate in humans and animal models of alimentary fat-induced insulin resistance, which is believed to be fundamental in the development of human type 2 diabetes.

We investigated whether long-term exposure of normal rats to a high-fat diet (HFD), inducing a type 2 diabetic phenotype, results in myocardial dysfunction and whether these alterations are associated with abnormal myocardial insulin signalling.

\section{Materials and methods}

Animals

The investigation conformed to the Guide for the Care and Use of Laboratory Animals as published by the NIH (NIH Publ. No. 85-23, revised 1996) and the regulations of the Institutional Animal Care and Use Committee. Adult male Wistar (WU) rats ( $n=34$; mean body weight $302 \pm 6 \mathrm{~g}$; Harlan CBP, Horst, The Netherlands) were fed an HFD for 7 weeks. Animals that received an isocaloric low-fat diet (LFD) for 7 weeks served as controls. At week 6, rats fasted for 6-8 $\mathrm{h}$ received an oral glucose load $(2 \mathrm{~g} / \mathrm{kg}$ of body weight). Blood glucose was measured from tail bleeds with a HemoCue glucose analyser (Angelholm, Sweden) at 0 , $15,30,60,90$ and $120 \mathrm{~min}$ after glucose ingestion. After 7 weeks, insulin stimulation was induced in fasted rats through i.p. injection of $10 \mathrm{U} / \mathrm{kg}$ body weight insulin (Actrapid $100 \mathrm{U} / \mathrm{ml}$; Novo Nordisk, The Netherlands). Similar insulin levels were achieved in HFD- and LFD-rats. Animals were considered insulin-stimulated when plasma insulin levels exceeded $400 \mu \mathrm{U} / \mathrm{ml}$. The effects of insulin were compared with those in animals that received a saline injection. Thirty minutes after saline or insulin injection, rats were killed by decapitation, and trunk blood was collected for glucose, insulin, leptin and NEFA determinations. Hearts were removed and transferred to ice-cold buffer containing (in mmol/l) $120 \mathrm{NaCl}, 1.22 \mathrm{MgSO}_{4}, 1.99$ $\mathrm{NaH}_{2} \mathrm{PO}_{4}, 27 \mathrm{NaHCO}_{3}, 15 \mathrm{KCl}, 1 \mathrm{CaCl}_{2}, 10$ glucose, and 30 2,3-butanedione 2-monoxime, equilibrated with $95 \% \mathrm{O}_{2}$ and $5 \% \mathrm{CO}_{2}$. Hearts were perfused via the aorta with the chilled buffer and a papillary muscle with part of the septum and septal artery was dissected from the right ventricle [10]. The remaining ventricular tissue was either snap-frozen in dry-ice-chilled isopentane and stored at $-80^{\circ} \mathrm{C}$ until analysis of insulin signalling, or fixed in Karnovky's universal fixative (EMS15720) for electron microscopy.

\section{Diets}

Experimental diets were obtained from Hope Farms (Woerden, The Netherlands; HFD: cat\#4148.02; LFD: cat\#4148.01). The LFD consisted of $8 \mathrm{wt} \%$ total fat, $22 \mathrm{wt} \%$ protein and $60 \mathrm{wt} \%$ carbohydrate; the HFD contained $25 \mathrm{wt}$ $\%$ fat, $32 \mathrm{wt} \%$ protein and $25 \mathrm{wt} \%$ carbohydrate, as well as more palmitate $(91.12 \mathrm{~g} / \mathrm{kg})$ and oleate $(100.24 \mathrm{~g} / \mathrm{kg}) \mathrm{com}$ pared with the LFD (29.12 and $32.08 \mathrm{~g} / \mathrm{kg}$, respectively). In the case of the HFD, $50.4 \%$ of the ingested calories were derived from fat compared with $16.4 \%$ for the LFD.

In vitro contractile function

Papillary muscles were mounted in a superfusion bath and connected to a force transducer to assess contractile function [10]. The septal artery was cannulated and perfused at $60 \mathrm{cmH}_{2} \mathrm{O}$. The muscle was superfused and perfused by buffer containing (in mmol/l) $118 \mathrm{NaCl}, 4.5 \mathrm{MgCl}_{2}, 4.5 \mathrm{KCl}$, $0.33 \mathrm{NaH}_{2} \mathrm{PO}_{4}, 25 \mathrm{NaHCO}_{3}, 1 \mathrm{CaCl}_{2}, 10$ glucose and 0.01 adenosine, equilibrated with $95 \% \mathrm{O}_{2}$ and $5 \% \mathrm{CO}_{2}$ and kept at $27^{\circ} \mathrm{C}$. Muscles were paced at $0.2 \mathrm{~Hz}$ and muscle dimensions were measured to enable normalisation of developed force $\left(F_{\mathrm{dev}}\right)$ and maximal force $\left(F_{\max }\right)$ on muscle crosssectional area. The length of the muscle was set at $95 \%$ of the maximal length as determined by a force-length relationship, followed by a stabilisation period of $60 \mathrm{~min}$. Muscles were subjected to a potentiation protocol to determine $F_{\max }$. A force-frequency protocol was applied to increase muscle workload and subsequently recovery of force $\left(F_{\text {rec }}\right)$ was determined, which is expressed as a percentage of initial force. Finally, to study the effect of $\mathrm{Ca}^{2+}$ on $F_{\text {dev }}$, muscles were exposed to $2 \mathrm{mmol} / \mathrm{l}$ extracellular $\mathrm{Ca}^{2+}$.

\section{Plasma determinations}

Insulin was measured by RIA (INSIK-5; DiaSorin Biomedica, Saluggia, Italy), which shows $100 \%$ cross-reactivity with rat insulin. Plasma NEFA levels were determined using a colorimetric kit (WAKO NEFA-C; Wako Pure Chemical Industries, Osaka, Japan). Serum leptin concentrations 
were measured by RIA (LEP-R61; Mediagnost, Reutlingen, Germany).

\section{Cardiac lipid analysis}

Triglyceride content in ventricular lysates was determined as described previously [11].

\section{Histology and electron microscopy}

Frozen sections from liver, skeletal muscle and cardiac ventricular tissue were stained with oil-red-O and counterstained with Mayer's haematoxylin. For electron microscopy, ultrathin sections of cardiac ventricular tissue were contrasted with uranyl acetate and Reynolds' lead citrate.

\section{Cardiac insulin signalling}

To determine insulin signalling, ventricular tissue was homogenised using an ultraturrax mixer [9]. Lysates were centrifuged $\left(15 \mathrm{~min} ; 14,000 \mathrm{rpm} ; 4^{\circ} \mathrm{C}\right)$, and protein content was determined using a BCA kit (Pierce, Rockford, IL, USA).

The insulin receptor $\beta$-subunit, IRS1, and IRS2 were immunoprecipitated using polyclonal insulin receptor $\beta$-subunit antibody C19 (Santa Cruz Biotechnology, Santa Cruz, CA, USA), IRS1 antibody K6, and IRS2 antibody K4, respectively [9]. Tyrosine phosphorylation of the immunoprecipitated proteins and IRS1/2-associated phosphatidylinositol 3'-kinase (PI3K) activity were determined as described [9]. Expression and phosphorylation of insulin signalling intermediates were analysed by Western blotting using anti-insulin receptor $\beta$-subunit (C19), anti-FOXO3/ FKHRL1 (H144), anti-phosphotyrosine-horseradish peroxidase conjugate, anti-endothelial nitric oxide synthase (eNOS; C20), anti-ryanodine receptor (RyR; N19) (Santa Cruz Biotechnology), anti-phospholamban (PLB), antiPLB-Ser16 (Upstate, Lake Placid, NY, USA), anti-FOXO1/ FKHR, anti-glycogen synthase kinase $3 \beta$ (GSK3 $\beta$ ), and phospho-specific protein kinase B (PKB)/Akt-Thr308, PKB/ Akt-Ser473, GSK3-Ser9/Ser21, eNOS-Ser1177, Forkhead/ FOXO-Thr24, and mammalian target of rapamycin (mTOR)Ser2448 antibodies (Cell Signaling Technology, Beverly, MA, USA). Polyclonal mTOR has been described [12], whereas PKB/Akt \#5178, and sarcoplasmic reticulum $\mathrm{Ca}^{2+}$ ATPase (SERCA2a) antibodies were kindly provided by Dr B. Burgering (UMC Utrecht, The Netherlands) and Dr W. S. Simonides (Laboratory for Physiology, VUMC, Amsterdam, The Netherlands), respectively. Immunoblots were quantified by densitometric analysis of the films.

\section{Statistical analysis}

Data are expressed as means \pm SD or means \pm SE. Differences between groups were determined by an unpaired Student's two-tailed $t$-test. A $p$ value of $p<0.05$ was considered statistically significant.

\section{Results}

HFD induces a non-obese phenotype with impaired glucose tolerance

Mean baseline body weights in LFD- and HFD-rats (302士 4.6 and $301 \pm 3.7 \mathrm{~g}$, respectively), and overall mean body weight gain during the course of the diet intervention (mean $49 \pm 4$ days; $132 \pm 31$ and $131 \pm 30 \mathrm{~g}$, respectively) were similar. Fasting $(p<0.001)$ and post-load blood glucose levels $(p<0.02)$ were significantly higher in HFD- than in LFDrats (Table 1, Fig. 1a). A significant short-term rise in postload plasma insulin levels was observed in HFD-rats only $(p<0.05$; Fig. 1b). At killing, no differences in plasma insulin, leptin and NEFA levels were found between the two groups (Table 1). Insulin injection suppressed plasma NEFA levels by $74.4 \%(p<0.001)$ in LFD-rats and by $26.7 \%$ ( $p=$ 0.111 ) in HFD-animals. Only the HFD induced skeletal muscle and liver steatosis (Fig. 1c-f).

HFD-feeding results in cardiac structural and functional abnormalities

Mean heart weight and heart-to-body-weight ratio of HFDrats were increased as compared with LFD-animals (both $p<0.01$; Table 1). Electron microscopy revealed marked ultrastructural abnormalities in cardiomyocytes from HFDrats, in particular degenerative changes of mitochondria, including matrix dilution, cristolysis and mitochondria-associated lamellar bodies (Fig. 2). Biochemical analysis showed a twofold rise in cardiac triglyceride content in HFD- vs LFD-rats $(p<0.02$; Table 1).

Table 1 Characteristics of rats after 7 weeks on a high- or low-fat diet

\begin{tabular}{|c|c|c|}
\hline & Low-fat diet & High-fat diet \\
\hline Body weight at killing $(\mathrm{g})$ & $458 \pm 27(n=16)$ & $461 \pm 29(n=18)$ \\
\hline $\begin{array}{l}\text { Fasting blood glucose } \\
(\mathrm{mmol} / \mathrm{l})\end{array}$ & $6.0 \pm 0.55(n=16)$ & $6.84 \pm 0.51(n=18)^{\mathrm{a}}$ \\
\hline $\begin{array}{l}\text { Fasting plasma insulin } \\
(\mathrm{pmol} / \mathrm{l})\end{array}$ & $398 \pm 97(n=8)$ & $315 \pm 83(n=5)$ \\
\hline Plasma leptin (mg/l) & $15.1 \pm 4.3(n=16)$ & $13.4 \pm 4.2(n=17)$ \\
\hline $\begin{array}{l}\text { Fasting plasma NEFA } \\
(\mathrm{mmol} / \mathrm{l})\end{array}$ & $0.39 \pm 0.11(n=6)$ & $0.30 \pm 0.07(n=6)$ \\
\hline Heart weight (g) & $1.96 \pm 0.13(n=15)$ & $2.16 \pm 0.20(n=15)^{\mathrm{b}}$ \\
\hline $\begin{array}{l}\text { (Heart weight:body } \\
\text { weight ratio }) \times 1,000\end{array}$ & $4.29 \pm 0.24(n=15)$ & $4.67 \pm 0.34(n=14)^{\mathrm{b}}$ \\
\hline $\begin{array}{l}\text { Cardiac triglycerides } \\
\text { ( } \mu \mathrm{g} / \mathrm{mg} \text { protein })\end{array}$ & $181 \pm 50(n=3)$ & $330 \pm 114(n=5)^{\mathrm{c}}$ \\
\hline
\end{tabular}

Values are means \pm SD

${ }^{\mathrm{a}} p<0.001$ vs low-fat

${ }^{\mathrm{b}} p<0.01$ vs low-fat

${ }^{c} p<0.02$ vs low-fat 
a
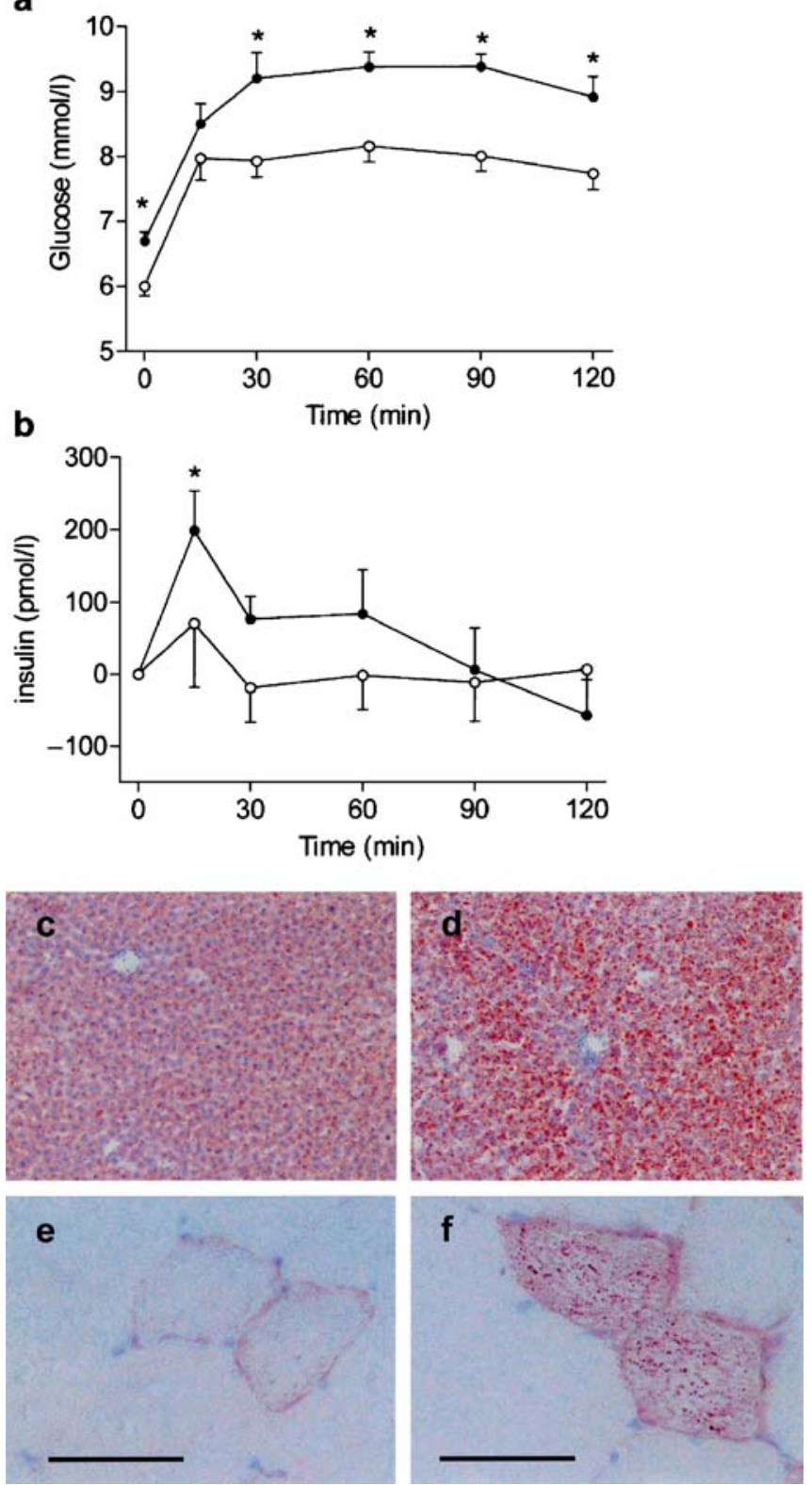

Fig. 1 Blood glucose levels following an oral glucose load in rats fed an HFD (black circles; $n=16$ ) and an LFD (white circles; $n=13)(\mathbf{a})$. Data are expressed as means $\pm \mathrm{SE}, * p<0.02$, diet effect. Baseline corrected plasma insulin levels following an oral glucose load in HFD(black circles; $n=5)$ and LFD-rats (white circles; $n=5)(\mathbf{b})$. Data are expressed as means $\pm \mathrm{SE}, * p<0.05$. Histological examination revealed lipid accumulation in the liver and skeletal muscle of HFD-rats ( $\mathbf{d}$ and f), but not in LFD-rats (c and e). Scale bar $=100 \mu \mathrm{m}$

Increases in basal $F_{\mathrm{dev}}$ and $F_{\max }$ of 1.7- $(p<0.01)$ and 1.6fold $(p<0.05)$, respectively were observed in papillary muscles obtained from saline-injected HFD-rats, as compared with those from LFD-rats (Fig. 3a, b). Similarly, $F_{\mathrm{dev}}$ showed a 1.4-fold increase $(p<0.05)$ in muscles from insulin-injected HFD- vs LFD-animals, but, when compared with the saline-injected groups, the stimulatory effect of insulin on $F_{\text {dev }}$ only reached significance in LFD-animals (Fig. 3a). $F_{\max }$ was not affected by insulin (Fig. $3 b$ ).

Following an enhanced workload, $F_{\text {rec }}$ equalled 69.1 and $65.8 \%$ of baseline $F_{\text {dev }}$ in saline- and insulin-injected LFD-
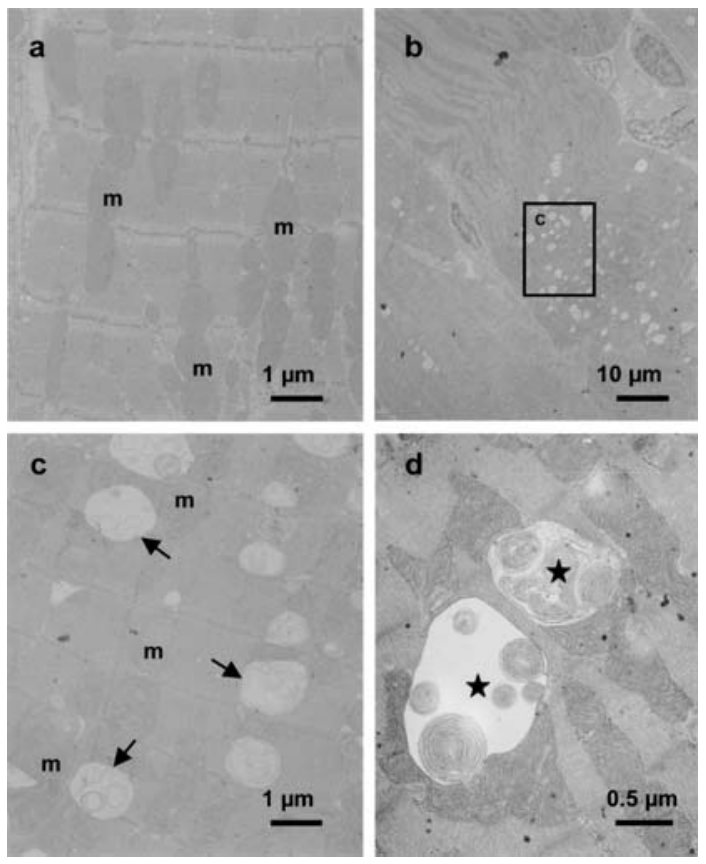

Fig. 2 Transmission electronmicrographs of cardiac sections of LFD- (a) and HFD-rats (b-d). Normal mitochondria $(m)$ with clearly visible cristae are present in LFD-cardiomyocytes, whereas degenerated mitochondria (arrow) are present in HFD-cells. Higher magnification revealed lamellar structures associated with mitochondrial membranes (star symbol)

animals, respectively (Fig. 3c), and 36.6 and $47.3 \%$ in the corresponding muscles from HFD-rats (both $p<0.05$ ), indicating an impaired recovery of HFD muscles. Increasing
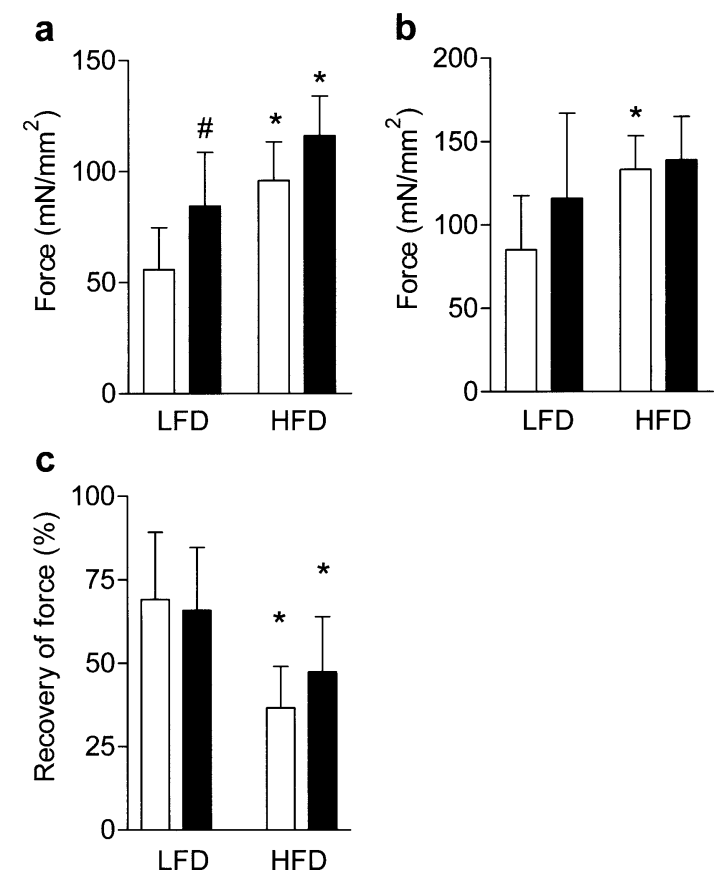

Fig. 3 Basal (a) and maximum (b) force development and recovery (c) after increased workload in papillary muscles from LFD- and HFD-fed rats (white bars $=$ saline; black bars $=$ insulin; $n=6$ in each condition). Data are expressed as means \pm SE, ${ }^{*} p<0.05$, diet effect; ${ }^{\#} p<0.05$, insulin effect 
the buffer $\mathrm{Ca}^{2+}$ concentration to $2 \mathrm{mmol} / \mathrm{l}$ induced a rise in $F_{\mathrm{dev}}$ in both LFD- and HFD-muscles from saline-injected rats (both $p<0.05$; Fig. 4 a). The $\mathrm{Ca}^{2+}$-induced $F_{\text {dev }}$ augmentation was blunted in muscles from insulin-injected LFD-rats $(p<0.05)$, but this insulin-mediated protective effect against $\mathrm{Ca}^{2+}$-overload was abolished in muscles from HFD-rats (Fig. 4a). These findings were not explained by differences in expression levels of sarcoplasmic reticulum $\mathrm{Ca}^{2+}$-cycling proteins RyR and SERCA2a (Fig. 4b). However, a $43 \%$ decrease in Ser16-PLB phosphorylation/PLB expression ratio was observed in hearts from insulin-injected HFD-rats ( $p<0.05$ vs LFD; Fig. $4 \mathrm{~b}, \mathrm{c})$, suggesting an increased inhibitory action of PLB on SERCA2a activity.

HFD reduces activation of multiple myocardial insulin signalling pathways

Phosphorylation of PKB/Akt and its substrates Insulin markedly increased both Thr308- and Ser473-PKB/Akt phosphorylation in LFD-hearts. PKB/Akt phosphorylation in HFD-hearts was reduced by $64(\mathrm{Thr} 308 ; p<0.02)$ and $45 \%$ (Ser473; $p<0.005)$ relative to $\mathrm{LFD}$, respectively (Fig. 5a, b). Protein expression of PKB/Akt was similar in LFD- and HFD-animals (Fig. 5a). Phosphorylation of the PKB/Akt-substrates eNOS-Ser1177, GSK3 $\beta$-Ser9 and FOXO1/3-Thr24 was increased in hearts from insulin-injected LFD-rats, but not in HFD-hearts (Fig. 5c). The blunted response to insulin of cardiac mTOR-Ser2448 phosphorylation in HFD- vs LFD-rats did not reach significance (Fig. 5c). Protein expression of the PKB/Akt-substrates did not differ among the experimental groups (Fig. 5d).

Tyrosine phosphorylation of IRS1/IRS2 and associated PI3K activity Insulin stimulation resulted in a 2.4-fold increase in IRS1 tyrosine phosphorylation in LFD-hearts (Fig. 6a, b), whereas the levels of tyrosine phosphorylated IRS1 were diminished by $57 \%$ in HFD-hearts $(p<0.001$; Fig. 6a, b). Furthermore, insulin induced a 4.7- vs 2.8-fold increase in IRS1-associated PI3K activity in LFD- vs HFDhearts $(p<0.02$; Fig. 6a, b). No difference was found in protein expression levels of IRS1 (Fig. 6c). The insulin-induced
Fig. 4 Prior insulin injection attenuated the $\mathrm{Ca}^{2+}$-induced increase in developed force $\left(F_{\mathrm{dev}}\right)$ in LFD-, but not in HFD-papillary muscles (a). Data are expressed as means $\pm \mathrm{SE}$ changes in $F_{\text {deqv }}$ after exposure to $2 \mathrm{mmol} / 1$ $\mathrm{Ca}^{2+}$, relative to $F_{\text {dev }}$ at $1 \mathrm{mmol} / 1$ $\mathrm{Ca}^{2+}$. Immunoblots (b) showing expression and/or phosphorylation of RyR, SERCA2a, PLB and PLB-Ser16. Quantification of PLB, PLB-Ser16 and the ratio PLB-Ser16:PLB (c). ${ }^{*} p<0.05$, diet effect; ${ }^{*} p<0.05$, insulin effect
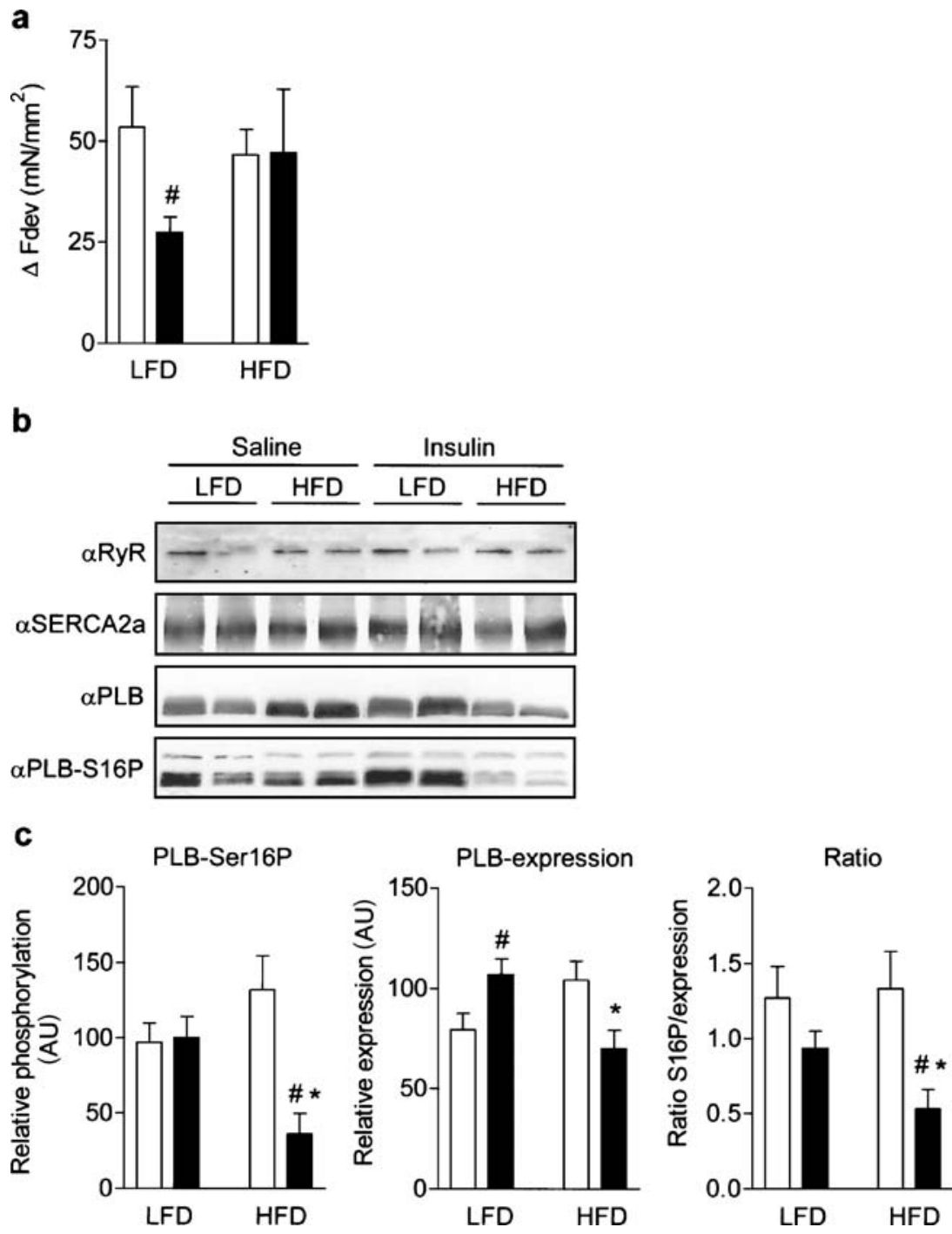


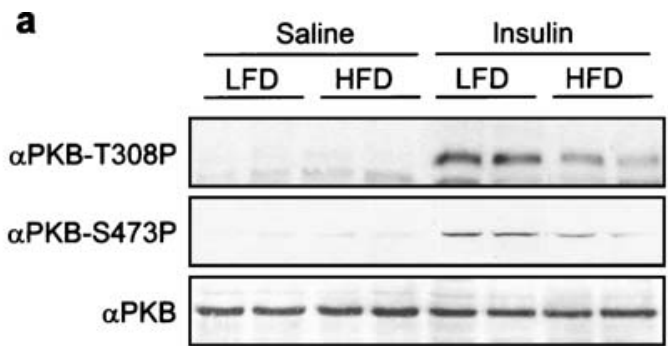

b

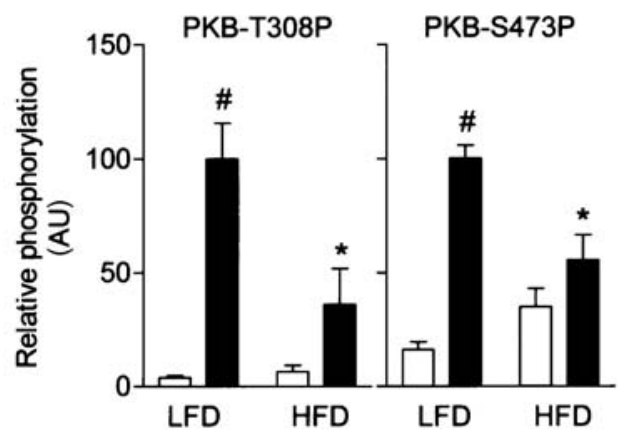

C
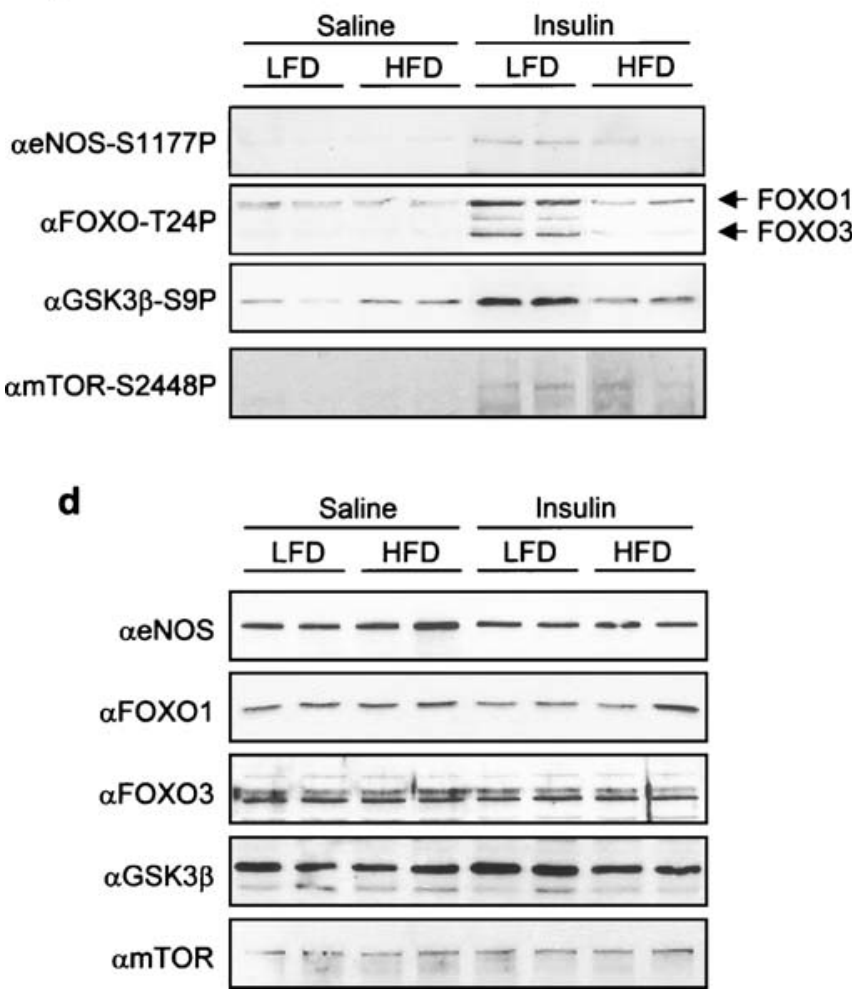

Fig. 5 Immunoblots (a) and the quantification (b) of PKB/AktThr308 and Ser473 phosphorylation (white bars=saline; black bars $=$ insulin; $n=8$ each condition). Immunoblots for phosphorylation (c) and expression (d) of eNOS, FOXO1/3, GSK3 $\beta$ and mTOR. Data are expressed as means $\pm \mathrm{SE},{ }^{*} p<0.05$, diet effect; ${ }^{*} p<0.05$, insulin effect

increase in tyrosine phosphorylation of IRS2 and IRS2-associated PI3K activity failed to reach significance under the experimental conditions used in this study (data not shown).
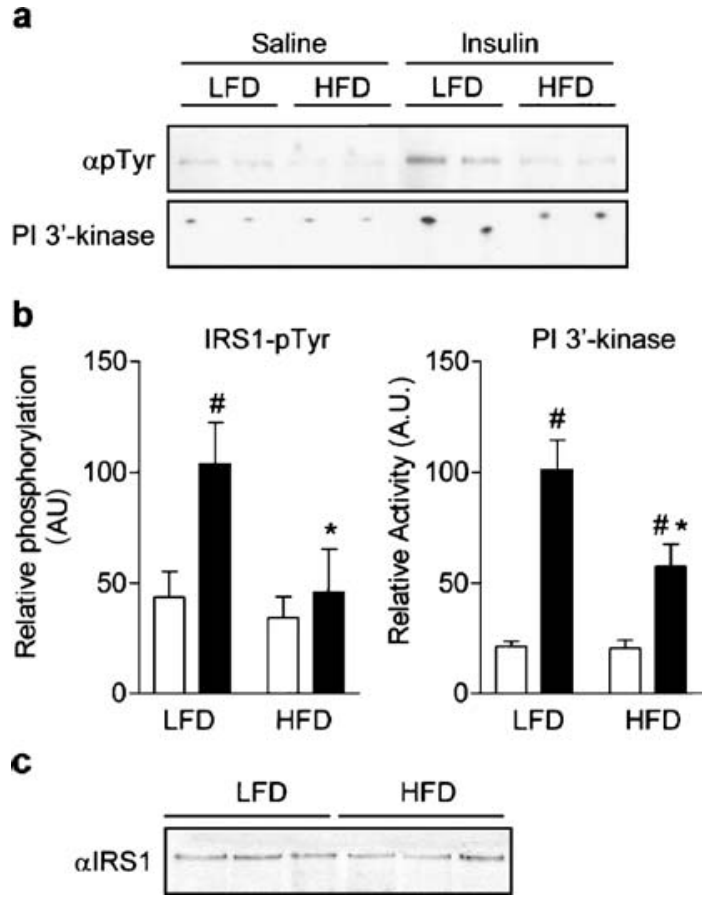

Fig. 6 Analysis (a) and quantification (b) of IRS1 immunoprecipitates for tyrosine phosphorylation (pTyr) and associated PI3K activity (white bars=saline; black bars=insulin). Immunoblot of IRS1 expression from saline-injected rats on an LFD and HFD (c). Data are expressed as means $\pm \mathrm{SE},{ }^{*} p<0.05$, diet effect; ${ }^{\#} p<0.05$, insulin effect

a

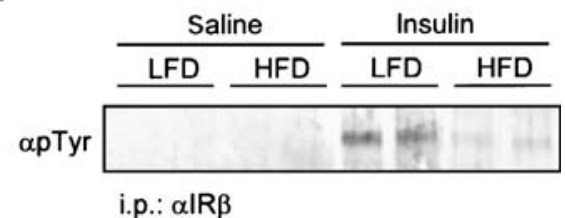

b

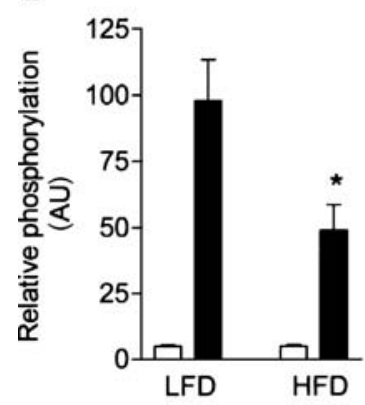

C

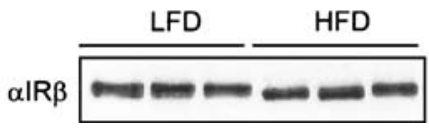

Fig. 7 Anti-phosphotyrosine (pTyr) immunoblot (a) and quantification (b) of insulin receptor $\beta$-subunit immunoprecipitates (white bars $=$ saline; black bars=insulin). Immunoblot of IR $\beta$-subunit expression from saline-injected rats on an LFD and HFD (c). Data are means \pm SE. ${ }^{*} p<0.05$, diet effect 
Tyrosine phosphorylation of the insulin receptor $\beta$-subunit Insulin induced tyrosine phosphorylation of the insulin receptor $\beta$-subunit in both groups (Fig. 7a). However, the response to insulin in HFD- vs LFD-rats was reduced by $50 \%(p<0.05$; Fig. $7 b)$. Expression of the insulin receptor protein was similar in both groups (Fig. 7c).

\section{Discussion}

Rats fed an HFD developed a type 2 diabetic phenotype, characterised by glucose intolerance and hepatic, skeletal muscle and cardiac steatosis, but with a body weight gain similar to that of LFD controls. HFD-feeding resulted in mild cardiac hypertrophy with higher baseline contractile forces but a markedly diminished recovery after higher workloads. Also, a blunted force-augmentation response to high $\mathrm{Ca}^{2+}$ was observed in insulin-injected HFD-rat papillary muscles, and cardiac ultrastructural abnormalities were found in the HFD-heart only. Furthermore, hearts from insulin-injected HFD-rats showed impaired insulin signalling and PLB hypophosphorylation.

So far, most studies investigating cardiac lipotoxicity have been performed in genetically manipulated rodents, particularly in leptin-deficient or -resistant animals [8, 13]. Therefore, it was unclear whether cardiac lipid accumulation could be induced by mere dietary HFD intake in normal animals. Normal rats fed a $60 \%$ HFD for 6 weeks had only a slight elevation of cardiac triglyceride content [7], whereas 10 -week-old leptin-deficient $o b / o b$ mice fed standard chow showed a three-fold increase in heart triglyceride content, relative to controls [13]. We found a two-fold increase in cardiac triglyceride content after 7 weeks of HFD-feeding in normal rats, indicating that chronic exposure to a high dietary fat content can indeed induce cardiac lipid accumulation and the associated abnormalities [14, 15].

The observed cardiac hypertrophy in the HFD-rats may be regarded as a first step in the sequence of adaptive responses of the heart to stress caused by a large number of physiological and pathological conditions [16]. Indeed, papillary muscles from the HFD-hearts showed higher basal and maximum forces, but a decreased recovery after higher workload. The underlying mechanism linking HFD-feeding to development of mild cardiac hypertrophy is unclear. On the one hand, hyperinsulinaemia has been implicated in the regulation of cardiac growth [17]. Alternatively, a contribution of triglyceride accumulation and sustained protein kinase $\mathrm{C}$ activation through triglyceride metabolites to the hypertrophic changes in HFD-hearts cannot be excluded [18]. Finally, although diet-related blood pressure elevations, contributing to the cardiac hypertrophy, cannot be entirely ruled out, the existence of relevant hypertension in our rats is less likely since no concentric hypertrophy was found (data not shown). Others have shown that blood pressure changes in rats given a 74\% fat-containing diet for 17 weeks were unremarkable [19].

Myocardial metabolic changes, i.e. increased NEFA oxidation at the expense of glucose uptake and metabolism, influence cardiac energy status and as such may have contributed to the observed functional alterations in HFDmuscles [20]. Although cardiac metabolism was not assessed, the observed signalling defects as well as the impaired capacity to recover from higher workloads are indicative of an impaired glucose metabolism in HFD-hearts. An increased NEFA supply will not only diminish the rate of glucose metabolism, but will also further increase oxygen consumption both by uncoupling oxidative phosphorylation [21] and by inducing cycling of NEFA in and out of the triglyceride pool, a futile, energy-consuming cycle [22]. Ischaemia is not likely to account for the observed changes since the muscle preparation was continuously perfused with oxygenated buffer [10]. In addition, high levels of NEFA may influence energy consumption related to excitationcontraction coupling as well as other processes related to cellular $\mathrm{Ca}^{2+}$ handling $[23,24]$. Therefore, the energetic disadvantage of the predominant use of NEFA as a myocardial energy substrate may result in the reduced ability to recover from a workload challenge.

Associations between myocardial metabolic abnormalities and dysfunction have been reported by some authors [24-27], but not by others [28, 29]. These discrepancies may be the result of differences in animal models and the methods used to assess cardiac function, including substrate availability and the presence or absence of myocardial ischaemia. Insulin may be an important link between myocardial metabolism and function [17, 30,31]; however, data on insulin-mediated effects and molecular aspects of insulin signalling are largely lacking from the aforementioned studies. Insulin improves cardiac energy efficiency by shifting substrate utilisation towards glucose, and in theory these changes may favourably affect myocardial function, in particular in the hypertrophic heart and under ischaemic conditions. These beneficial effects of insulin were shown both in animals [20], and in humans [32].

HFD-papillary muscles displayed functional insulin resistance, as reflected by the absence of positive inotropy and impaired protection against $\mathrm{Ca}^{2+}$ overload. An altered myocardial contractile response to insulin was previously described in models of diabetes and cardiac hypertrophy [33-37]. Alterations in cardiomyocyte $\mathrm{Ca}^{2+}$ handling in streptozotocin-induced diabetic hearts have been linked to decreased expression and activation of SERCA2a, amongst others through interaction with IRS1/2 [38, 39]. We found no effect of insulin on SERCA2a-IRS1/2 association (data not shown), and, in line with other studies in animal models of insulin resistance/type 2 diabetes, no diet-induced alterations in SERCA2a and RyR expression [40, 41]. However, the observed decrease in Ser16-PLB phosphorylation levels in insulin-injected HFD-hearts may have contributed to the impaired insulin-mediated protection against $\mathrm{Ca}^{2+}$ overload, as a reduction in Ser16-PLB phosphorylation increases the inhibitory action of PLB on SERCA2a activity [42].

At the molecular level, insulin resistance in HFD-hearts was reflected by impaired activation of the insulin receptor and IRS1/PI3K/PKB/Akt-mediated signalling. Although studies regarding insulin receptor activation in the skeletal muscle of type 2 diabetic subjects have yielded conflicting results [43], defects in insulin receptor activation were 
reported in skeletal muscle from HFD-rats [44] as well as in hearts from leptin-deficient rodents [45, 46]. Various studies have linked NEFA metabolites, like ceramides and diacylglycerol, to activation of protein kinase C, Jun Nterminal kinase, and inhibitor of nuclear factor- $\mathrm{kB}$ kinase, which in their turn can abrogate insulin signalling through serine phosphorylation of the insulin receptor and its substrates [14]. We found triglyceride accumulation in skeletal and cardiac muscle from HFD-rats in the presence of unaltered circulating NEFA levels, suggesting that an increased supply of NEFA originating from intramyocellular triglyceride stores, rather than elevated circulating NEFA levels, could be responsible for the observed cardiac insulin resistance. Furthermore, activation of protein phosphatases contributing to impaired insulin signalling in HFD-hearts cannot be ruled out, since preliminary data indicate a slight elevation of protein phosphatase $2 \mathrm{~A}$ expression in HFD-hearts (data not shown), which has been implicated in the dephosphorylation of, amongst others, PKB/Akt and PLB [47, 48].

The decreased activation of PKB/Akt-mediated signalling in HFD-hearts may result not only in decreased GLUT4mediated glucose uptake and glycogen synthesis [14], but also in adverse effects on cardiac function. Reduced phosphorylation of eNOS may impair nitric oxide generation and vasodilatation, thereby adversely affecting cardiac function [49]. Finally, here we report for the first time the insulin-mediated phosphorylation of the forkhead transcription factors FOXO1/3 in the heart. Phosphorylation of the forkhead transcription factors FOXO1/3 by PKB/Akt results in their nuclear exclusion and concurrent loss of their gene regulatory function [50]. Although in vitro studies implicate FOXO proteins in cell cycle control, cell survival and apoptosis, their function in the heart clearly depends on future target gene identification.

Taken together, an HFD induced a type 2 diabetic phenotype in rats, cardiac dysfunction and both functional and molecular myocardial insulin resistance. Insulin exerts many different effects that collectively may, directly or indirectly, have a beneficial impact on the heart. However, it still remains to be demonstrated whether insulin can effect these additional actions independently of its metabolic effects. Interventions aimed at ameliorating cardiac insulin signalling may constitute a major therapeutic contribution in the battle against diabetes-related heart disease.

Acknowledgements The authors wish to thank Dr H. Niessen (Department of Pathology, VU University Medical Centre, Amsterdam, The Netherlands) for helpful discussions, and Gerard van der Zon (Department of Molecular Cell Biology, LUMC, The Netherlands) and Maarten Tushuizen (Department of Endocrinology, VU University Medical Centre, Amsterdam, The Netherlands) for technical assistance.

\section{References}

1. Kannel WB, Hjortland M, Castelli WP (1974) Role of diabetes in congestive heart failure: the Framingham study. Am J Cardiol 34:29-34
2. Stamler J, Vaccaro O, Neaton JD, Wentworth D (1993) Diabetes, other risk factors, and 12-yr cardiovascular mortality for men screened in the Multiple Risk Factor Intervention Trial. Diabetes Care 16:434-444

3. Vanninen E, Mustonen J, Vainio P, Lansimies E, Uusitupa M (1992) Left ventricular function and dimensions in newly diagnosed non-insulin-dependent diabetes mellitus. Am J Cardiol 70:371-378

4. Diamant M, Lamb HJ, Groeneveld Y et al (2003) Diastolic dysfunction is associated with altered myocardial metabolism in asymptomatic normotensive patients with well-controlled type 2 diabetes mellitus. J Am Coll Cardiol 42:328-335

5. Bell DS (1995) Diabetic cardiomyopathy. A unique entity or a complication of coronary artery disease? Diabetes Care 18:708 714

6. Taegtmeyer H, McNulty P, Young ME (2002) Adaptation and maladaptation of the heart in diabetes: part I. General concepts. Circulation 105:1727-1733

7. Unger RH (2002) Lipotoxic diseases. Annu Rev Med 53:319336

8. Zhou YT, Grayburn P, Karim A et al (2000) Lipotoxic heart disease in obese rats: implications for human obesity. Proc Natl Acad Sci U S A 97:1784-1789

9. Kessler A, Uphues I, Ouwens DM, Till M, Eckel J (2001) Diversification of cardiac insulin signaling involves the $\mathrm{p} 85 \mathrm{alph} /$ beta subunits of phosphatidylinositol 3-kinase. Am J Physiol Endocrinol Metab 280:E65-E74

10. Lamberts RR, Van Rijen MH, Sipkema P, Fransen P, Sys SU, Westerhof N (2002) Coronary perfusion and muscle lengthening increase cardiac contraction: different stretch-triggered mechanisms. Am J Physiol Heart Circ Physiol 283:H1515-H1522

11. Bligh EG, Dyer WJ (1959) A rapid method of total lipid extraction and purification. Can J Med Sci 37:911-917

12. Withers DJ, Ouwens DM, Nave BT et al (1997) Expression, enzyme activity, and subcellular localization of mammalian target of rapamycin in insulin-responsive cells. Biochem Biophys Res Commun 241:704-709

13. Christoffersen C, Bollano E, Lindegaard MLS et al (2003) Cardiac lipid accumulation associated with diasystolic dysfunction in obese mice. Endocrinology 144:3483-3490

14. Perseghin G, Petersen K, Shulman GI (2003) Cellular mechanism of insulin resistance: potential links with inflammation. Int J Obes Relat Metab Disord 27(Supp1 3):S6-S11

15. Schaffer JE (2003) Lipotoxicity: when tissues overeat. Curr Opin Lipidol 14:281-287

16. Frey N, Katus HA, Olson EN, Hill JA (2004) Hypertrophy of the heart: a new therapeutic target? Circulation 109:1580-1589

17. Belke DD, Betuing S, Tuttle MJ et al (2002) Insulin signaling coordinately regulates cardiac size, metabolism, and contractile protein isoform expression. J Clin Invest 109:629-639

18. Murphy S, Frishman WH (2005) Protein kinase C in cardiac disease and as a potential therapeutic target. Cardiol Rev 13:3-12

19. Fitzgerald SM, Henegar JR, Brands MW, Henegar LK, Hall JE (2001) Cardiovascular and renal responses to a high-fat diet in Osborne-Mendel rats. Am J Physiol Regul Integr Comp Physiol 281:R547-R552

20. Korvald C, Elvenes OP, Myrmel T (2000) Myocardial substrate metabolism influences left ventricular energetics in vivo. Am J Physiol Heart Circ Physiol 278:H1345-H1351

21. Borst P, Loos JA, Christ EJ, Slater EC (1962) Uncoupling activity of long-chain fatty acids. Biochim Biophys Acta 62:509518

22. Myrmel T, Forsdahl K, Larsen TS (1992) Triacylglycerol metabolism in hypoxic, glucose-deprived rat cardiomyocytes. J Mol Cell Cardiol 24:855-868

23. Philipson KD, Ward R (1985) Effects of fatty acids on $\mathrm{Na}^{+}-\mathrm{Ca}^{2+}$ exchange and $\mathrm{Ca}^{2+}$ permeability of cardiac sarcolemmal vesicles. J Biol Chem 260:9666-9671

24. Huang JM, Xian H, Bacaner M (1992) Long-chain fatty acids activate calcium channels in ventricular myocytes. Proc Natl Acad Sci U S A 89:6452-6456 
25. Chiu HC, Kovacs A, Ford DA et al (2001) A novel mouse model of lipotoxic cardiomyopathy. J Clin Invest 107:813-822

26. Finck BN, Lehman JJ, Leone TC et al (2002) The cardiac phenotype induced by PPARalpha overexpression mimics that caused by diabetes mellitus. J Clin Invest 109:121-130

27. Young ME, Guthrie PH, Razeghi P et al (2002) Impaired longchain fatty acid oxidation and contractile dysfunction in the obese Zucker rat heart. Diabetes 51:2587-2595

28. Abel ED, Kaulbach HC, Tian R et al (1999) Cardiac hypertrophy with preserved contractile function after selective deletion of GLUT4 from the heart. J Clin Invest 104:1703-1714

29. Atkinson LL, Kozak R, Kelly SE, Onay BA, Russell JC, Lopaschuk GD (2003) Potential mechanisms and consequences of cardiac triacylglycerol accumulation in insulin-resistant rats. Am J Physiol Endocrinol Metab 284:E923-E930

30. Hu P, Zhang D, Swenson L, Chakrabarti G, Abel ED, Litwin SE (2003) Minimally invasive aortic banding in mice: effects of altered cardiomyocyte insulin signaling during pressure overload. Am J Physiol Heart Circ Physiol 285:H1261-H1269

31. Dutta K, Podolin DA, Davidson MB, Davidoff AJ (2001) Cardiomyocyte dysfunction in sucrose-fed rats is associated with insulin resistance. Diabetes 50:1186-1192

32. Fath-Ordoubadi F, Beatt KJ (1997) Glucose-insulin-potassium therapy for treatment of acute myocardial infarction: an overview of randomized placebo-controlled trials. Circulation 96:11521156

33. Ren J, Walsh MF, Hamaty M, Sowers JR, Brown RA (1999) Augmentation of the inotropic response to insulin in diabetic rat hearts. Life Sci 65:369-380

34. Ren J, Sowers JR, Walsh MF, Brown RA (2000) Reduced contractile response to insulin and IGF-I in ventricular myocytes from genetically obese Zucker rats. Am J Physiol Heart Circ Physiol 279:H1708-H1714

35. Allard MF, Wambolt RB, Longnus SL et al (2000) Hypertrophied rat hearts are less responsive to the metabolic and functional effects of insulin. Am J Physiol Endocrinol Metab 279:E487-E493

36. Doenst T, Goodwin GW, Cedars AM, Wang M, Stepkowski S, Taegtmeyer H (2001) Load-induced changes in vivo alter substrate fluxes and insulin responsiveness of rat heart in vitro. Metabolism 50:1083-1090

37. Sasso FC, Carbonara O, Cozzolino D et al (2000) Effects of insulin-glucose infusion on left ventricular function at rest and during dynamic exercise in healthy subjects and noninsulin dependent diabetic patients: a radionuclide ventriculographic study. J Am Coll Cardiol 36:219-226
38. Netticadan T, Temsah RM, Kent A, Elimban V, Dhalla NS (2001) Depressed levels of $\mathrm{Ca}^{2+}$-cycling proteins may underlie sarcoplasmic reticulum dysfunction in the diabetic heart. Diabetes 50:2133-2138

39. Algenstaedt P, Antonetti DA, Yaffe MB, Kahn CR (1997) Insulin receptor substrate proteins create a link between the tyrosine phosphorylation cascade and the $\mathrm{Ca}^{2+}$-ATPases in muscle and heart. J Biol Chem 272:23696-23702

40. Dutta K, Carmody MW, Cala SE, Davidoff AJ (2002) Depressed PKA activity contributes to impaired SERCA function and is linked to the pathogenesis of glucose-induced cardiomyopathy. J Mol Cell Cardiol 34:985-996

41. Belke DD, Swanson EA, Dillmann WH (2004) Decreased sarcoplasmic reticulum activity and contractility in diabetic $d b$ $d b$ mouse heart. Diabetes 53:3201-3208

42. MacLennan DH, Kranias EG (2003) Phospholamban: a crucial regulator of cardiac contractility. Nat Rev Mol Cell Biol 4:566577

43. Zierath JR, Krook A, Wallberg-Henriksson H (2000) Insulin action and insulin resistance in human skeletal muscle. Diabetologia 43:821-835

44. Youngren JF, Paik J, Barnard RJ (2001) Impaired insulin-receptor autophosphorylation is an early defect in fat-fed, insulinresistant rats. J Appl Physiol 91:2240-2247

45. Mazumder PK, O’Neill BT, Roberts MW et al (2004) Impaired cardiac efficiency and increased fatty acid oxidation in insulinresistant $o b / o b$ mouse hearts. Diabetes 53:2366-2374

46. Carvalheira JB, Calegari VC, Zecchin HG et al (2003) The cross-talk between angiotensin and insulin differentially affects phosphatidylinositol 3-kinase- and mitogen-activated protein kinase-mediated signaling in rat heart: implications for insulin resistance. Endocrinology 144:5604-5614

47. Cazzolli R, Carpenter L, Biden TJ, Schmitz-Peiffer C (2001) A role for protein phosphatase $2 \mathrm{~A}$-like activity, but not atypical protein kinase Czeta, in the inhibition of protein kinase $\mathrm{B} / \mathrm{Akt}$ and glycogen synthesis by palmitate. Diabetes 50:2210-2218

48. Gergs U, Boknik P, Buchwalow I et al (2004) Overexpression of the catalytic subunit of protein phosphatase $2 \mathrm{~A}$ impairs cardiac function. J Biol Chem 279:40827-40834

49. Gao F, Gao E, Yue TL et al (2002) Nitric oxide mediates the antiapoptotic effect of insulin in myocardial ischemia-reperfusion: the roles of PI3-kinase, Akt, and endothelial nitric oxide synthase phosphorylation. Circulation 105:1497-1502

50. Accili D, Arden KC (2004) FoxOs at the crossroads of cellular metabolism, differentiation, and transformation. Cell 117:421426 